\title{
DETERMINAÇÃO DE GINKGOFLAVONÓIDES POR CROMATOGRAFIA LÍQUIDA DE ALTA EFICIÊNCIA EM MATÉRIAS- PRIMAS E PRODUTOS ACABADOS
}

\section{Determination of Ginkgo flavonoids in raw material and pharmaceutical products by high performance-UV liquid chromatography}

\author{
Maria T. F. Bara ${ }^{1,2^{*}}$; Hérica N. Cirilo ${ }^{1,3}$; Valéria de Oliveira ${ }^{1,4}$ \\ ${ }^{1}$ Laboratório de Controle de Qualidade de Medicamentos, Faculdade de Farmácia, Universidade Federal de Goiás. \\ Praça Universitária s/n. 74605-220. Goiânia-GO, Brasil. \\ ${ }^{2}$ Profa da disciplina de Farmacognosia da FF/UFG; \\ ${ }^{3}$ Farmacêutica responsável pelo Controle de Qualidade Físico-Químico do LCQM/UFG; \\ ${ }^{4}$ Prof $^{a}$ de Química Farmacêutica da FF/UFG.
}

*Autor para correspondência e-mail: mbara@farmacia.ufg.br

Recebido em 05/10/2004 - Aceito em 16/11/2004

\begin{abstract}
RESUMO: Produtos farmacêuticos a base de Ginkgo biloba L. constituem o medicamento fitoterápico de maior comercialização no Brasil. Ensaios clínicos e farmacológicos com extrato EGb761 tem demonstrado que a sua principal indicação terapêutica é nos casos de insuficiência circulatória periférica e insuficiência cerebrovascular. Os compostos ativos são flavonóides e lactonas terpênicas. Neste trabalho foi avaliada a qualidade de amostras de extratos secos e de cápsulas de Ginkgo. O método para a determinação de ginkgoflavonóides foi CLAE e os resultados demonstraram ser um método simples, rápido e adequado para a análise destes produtos farmacêuticos. Verificou-se que $33 \%$ e 91\%, respectivamente, da matéria-prima e das cápsulas estavam em desacordo com as especificações declaradas pelos fabricantes nas análises de controle de qualidade, em relação ao seu teor de ginkgoflavonóides. Os dados obtidos mostraram ser fundamental a implementação do controle de qualidade de plantas medicinais e de fitoterápicos.
\end{abstract}

PALAVRAS-CHAVE: Ginkgo biloba, controle de qualidade, fitoterápico, matéria-prima vegetal, cromatografia líquida.

\begin{abstract}
Pharmaceutical preparations containing Ginkgo biloba L. have large market. Pharmacological and clinical survey on EGb761 extract have demonstrated application on peripheral circulatory and cerebrovascular insufficiency. The Ginkgo extracts contain as active compounds flavonoids and terpene lactones. In this work the quality of the standardization extracts and of the preparations containing Ginkgo was evaluated. The method for quantification of Ginkgo flavonoids was liquid chromatography and data showed to be simple, rapid and adequate to this analysis. The analytical results demonstrated that $33 \%$ and $92 \%$, respectively, plant extract and capsule were not in agreement with the specifications. This analysis showed valuable for herbal medicines and phytomedicines quality control.
\end{abstract}

KEYWORDS: Ginkgo biloba, quality control, phytomedicine, liquid chromatography.

\section{INTRODUÇÃO}

Os extratos de Ginkgo biloba L. contêm flavonóides como substâncias ativas, com cerca de 21 já isolados. Dentre estes uma grande variedade de glicosídeos de núcleo flavonol como quercetina, campferol e isoramnetina 
(Fig. 1). Ocorrem também nestes extratos lactonas terpênicas, como ginkgolídeos e bilobalídeos, porém devido às baixas concentrações na planta, às fracas características de absorção na região do UV e às dificuldades na etapa de extração, estes não têm sido usado na padronização de extrato de Ginkgo (STICHER, 1993).

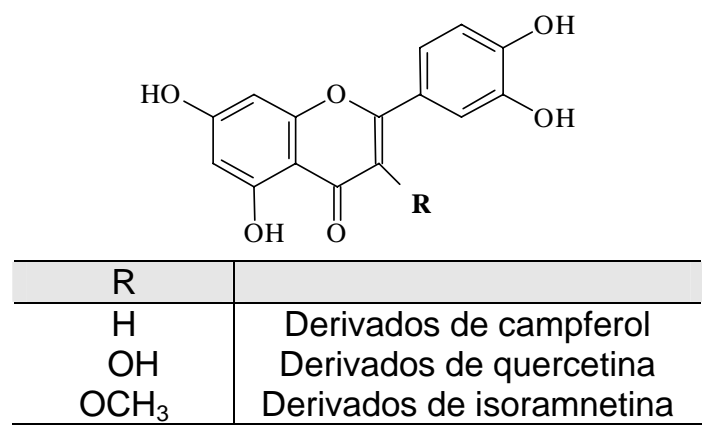

Figura 1. Estrutura de algumas agliconas de flavonóides presentes nas folhas de Ginkgo biloba L.

Para quantificar os ginkgoflavonóides, tem sido usado o método CLAE (USP 25, 2002; STICHER, 1993), que preconizam inicialmente uma extração e hidrólise dos glicosídeos em meio contendo ácido. As agliconas libertadas dos flavonóides podem ser facilmente analisadas por CLAE em fase reversa usando um gradiente metanol-água com 0,3-0,5\% v/v de ácido fosfórico e detector UV ajustado em $370 \mathrm{~nm}$. O perfil cromatográfico característico do extrato de ginkgo hidrolisado é mostrado no cromatograma (Fig 2). Observa-se a quercetina como um dos principais componentes e isoramnetina é eluída aproximadamente 10 min após.

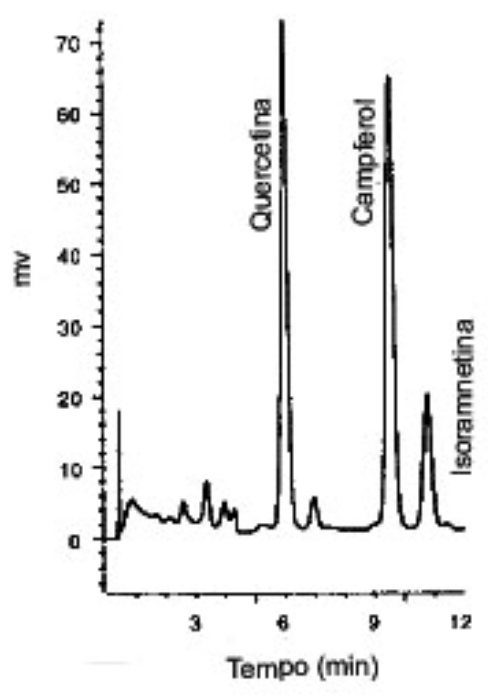

Figura 2. Cromatograma obtido por CLAE de extrato de Ginkgo biloba após hidrólise dos flavonóides (STICHER, 1993).

O desenvolvimento de metodologias por meio de cromatografia gasosa acoplada a espectrometria de massa (CG-EM) para análise de extrato padronizado e cápsulas de Ginkgo constitui outro método analítico descrito na literatura (DENG \& ZITO, 2003). Também têm sido descritos métodos para determinação de quercetina e campferol por CLAE (WANG, et al, 2003) ou por CG-EM (WATSON \& PITT, 1998; WATSON \& OLIVEIRA, 1999) em urinas de humanos submetidos a tratamentos com comprimidos de Ginkgo, os quais são excretados na forma de glicuronídeos.

O extrato padronizado de Ginkgo biloba EGb761, segundo a RDC 89/2004 (BRASIL, 2004), deve conter no mínimo $24,0 \%$ de ginkgoflavonóides e $6 \%$ de terpenóides. As cápsulas de ginkgo disponíveis no mercado freqüentemente são preparadas na concentração de 40, 80 ou $120 \mathrm{mg}$ de flavonóides. O uso de pó das folhas desta planta não é recomendado por apresentar ácido gincólico, substância alergênica que é encontrada em concentrações menores que 5 ppm no extrato padronizado. Além disso, sua concentração em flavonóides é 
aproximadamente de 0,8\%. Ginkgo biloba constitui o fitoterápico mais comercializado no Brasil (SUZUKI, 2002), sendo usado por ter um mecanismo de ação polivalente: neuroproteção, vaso-regulação generalizada, antioxidante, sequestrador de radicais livres (SIMÕES \& FARIAS, 2002). As reações adversas mais freqüentes incluem náusea, azia, dor de cabeça, hematoma subdural e hemorragia subaracnóide intracerebral (DESAI \& GROSSBERG, 2003).

Embora diversos estudos tenham procurado comprovar a eficácia e garantir segurança aos produtos de origem vegetal (BAUER \& TITTEL, 1996; CHOI, et al, 2002; BAST, et al 2002), o desenvolvimento, aplicação e a validação de métodos analíticos para matérias-primas e fitoterápicos ainda são escassos na literatura (STICHER, 1993; WATSON \& PITT, 1998; VAN BEEK \& WINTERMANS, 2001). Estudos também devem ser realizados visando determinar parâmetros de dissolução e de biodisponibilidade dos componentes ativos vegetais, como demonstrado para comprimidos e cápsulas de Ginkgo biloba (KRESSMANN, et al, 2002).

Acredita-se que a aplicação de rígidas especificações de controle de qualidade pode acarretar um maior desenvolvimento da indústria farmacêutica de fitoterápicos e fitofármacos no Brasil (YUNES, et al, 2001). O mercado farmacêutico destes produtos é amplo, no entanto, monografias ainda são escassas na Farmacopéia Brasileira. Deste modo, a necessidade de monitorar o teor de ginkgoflavonóides, tanto na matéria prima, como em fitoterápicos motivou a avaliação da qualidade de produtos à base de Ginkgo biloba.

\section{MATERIAL E MÉTODOS}

\section{Reagentes}

Metanol grau UV/HPLC-espectroscópico $\left(V_{\text {etec }}{ }^{R}\right)$ e ácido fosfórico 85\% (Synth $\left.{ }^{R}\right)$ foram usados no preparo da fase móvel. A água usada nas análises cromatográficas foi submetida em sistema Milli-Q (Millipore). Metanol grau analítico $\left(\right.$ Vetec $^{R}$ ) foi usado no preparo das amostras. Quercetina dihidratada (Sigma ${ }^{R}$ ), numa solução contendo $0,05 \mathrm{mg} \mathrm{ml}^{-1}$ em metanol foi utilizada como padrão externo.

\section{Cromatógrafo líquido e condições experimentais}

As análises foram realizadas em cromatógrafo líquido Varian, modelo ProStar, acoplado com detector UV, modelo ProStar (Varian), software Varian Star Workstation versão 5.0 foi usado para registrar os cromatogramas e medir as áreas dos picos. A separação dos ginkgoflavonóides foi executada usando uma coluna Microsorb-MV $100 \mathrm{C}_{18}(250 \mathrm{~mm} \times 4,6 \mathrm{~mm} \times 5 \mu \mathrm{m})$. Os solventes foram previamente filtrados em membrana de $0,45 \mu \mathrm{m}$ (Millipore) e então desgazeificados em banho ultrassônico (Ultrasonic Cleaner Unique). Cromatografia foi realizada à temperatura de $25^{\circ} \mathrm{C}$. A eluição foi realizada com metanol / água-ácido fosfórico 0,3\% (v/v) num fluxo de $1 \mathrm{ml} \mathrm{min} \mathrm{m}^{-1}$. O gradiente inicial foi 30\% A - $70 \%$ B; final foi 60\% A; 40\% B. Alíquotas de $20 \mu \mathrm{l}$ das amostras foram injetadas com seringa Hamilton de $50 \mu \mathrm{l}$ e a detecção dos picos foi registrada a $370 \mathrm{~nm}$. Tempo de corrida foi de $40 \mathrm{~min}$. Os cálculos de teor de ginkgoflavonóides foi baseado num fator de conversão das agliconas em glicosídeos, que para a quercetina é de =2,504, para campferol é de 2,64 e para a isorahmnetina é de 2,437.

$$
\sum \text { (agliconas) } \times 2,51 \text { = conteúdo de ginkgoflavonóides }
$$

\section{Produtos analisados}

Foram analisados produtos de 05 fornecedores, compondo 10 lotes, totalizando 15 amostras de extratos secos padronizados de Ginkgo biloba e cápsulas de 11 empresas, compondo 11 lotes, cujo rótulo declarava conter 80 mg de ginkgoflavonóides.

\section{Preparo da amostra}

Foram pesados, exatamente, $200 \mathrm{mg}$ dos extratos secos de Ginkgo biloba que foram solubilizados em 50 $\mathrm{mL}$ de metanol $80 \%$ em agitador Wrist Action modelo 75 por 5 minutos. Uma alíquota de $10 \mathrm{ml}$ desta solução foi colocada em balão de fundo chato de $125 \mathrm{~mL}$ e adicionada de $10 \mathrm{~mL}$ de uma solução de metanol $80 \%$ acrescentado de $5,5 \%$ de $\mathrm{HCl}$. O material foi colocado em banho Maria $85^{\circ} \mathrm{C}$ por 2 horas para a hidrólise dos glicosídeos. Após esfriar, filtrou-se em papel (Inlab tipo 30) e completou-se o volume para $25 \mathrm{~mL}$ com metanol $80 \%$, em balão volumétrico. Finalmente filtrou-se esta solução contendo os ginkgoflavonóides em filtro Millex (Milipore $^{\mathrm{R}}$ ) 0,45 $\mu \mathrm{m}$ e analisou-se por CLAE.

Para as cápsulas, inicialmente calculou-se o peso médio (FARMACOPÉIA BRASILEIRA, 1988) e calculouse a quantidade correspondente a $200 \mathrm{mg}$ de extrato seco. 


\section{RESULTADOS E DISCUSSÃO}

A análise do teor dos principais componentes biologicamente ativos em matérias primas de origem vegetal e/ou de fitoterápicos é uma etapa essencial para a segurança e eficácia de sua utilização na elaboração de produtos farmacêuticos. A quantificação de substâncias ativas em fitoterápicos ainda é realizada de forma incipiente devido a presença de um fitocomplexo ativo nas plantas e seus extratos (WILLIAMSON, 2001), o que dificulta a sua análise. Além disso, a disponibilidade de um número reduzido de substâncias químicas de referência para comparação com matérias primas vegetais, que raramente apresentam a substância química isolada contribui para esta situação. Mediante este quadro, o uso de extratos padronizados centrados em grupos específicos de princípios ativos visa garantir a homogeneidade química do produto lote a lote, para melhoria da qualidade (BAUER, 1998; CAPASSO, et al., 2000). Os princípios ativos selecionados para esse processo de ajuste de qualidade devem ser aqueles que são importantes para as ações biológica e farmacológica do produto, se conhecidos (CALIXTO, 2000).

O extrato padronizado de Ginkgo biloba EGb761 disponível no mercado apresenta segundo as especificações do fabricante, um teor de 22,0 a 27,0\% de flavonóides, 5,0 a 7,0\% de lactonas terpênicas e menos de 5 ppm de ácidos gincólicos. $O$ pó de ginkgo não tem equivalência farmacêutica com o extrato, sendo que 100 $\mathrm{kg}$ folha corresponderiam ao uso de 2,5 kg extrato (LOEW, 1997; SIMÕES \& FARIAS, 2002).

Os resultados obtidos neste estudo estão apresentados na Tabela 1 e demonstraram a necessidade do desenvolvimento e implementação do controle de qualidade de matérias primas vegetais e de fitoterápicos à base de ginkgo. Conforme Tabela 1 e Figura 3, verificou-se que 33,0\% e 91,0\%, respectivamente, da matéria-prima e das cápsulas analisadas não corresponderam aos valores declarados pelo fabricante em relação ao seu teor de ginkgoflavonóides.

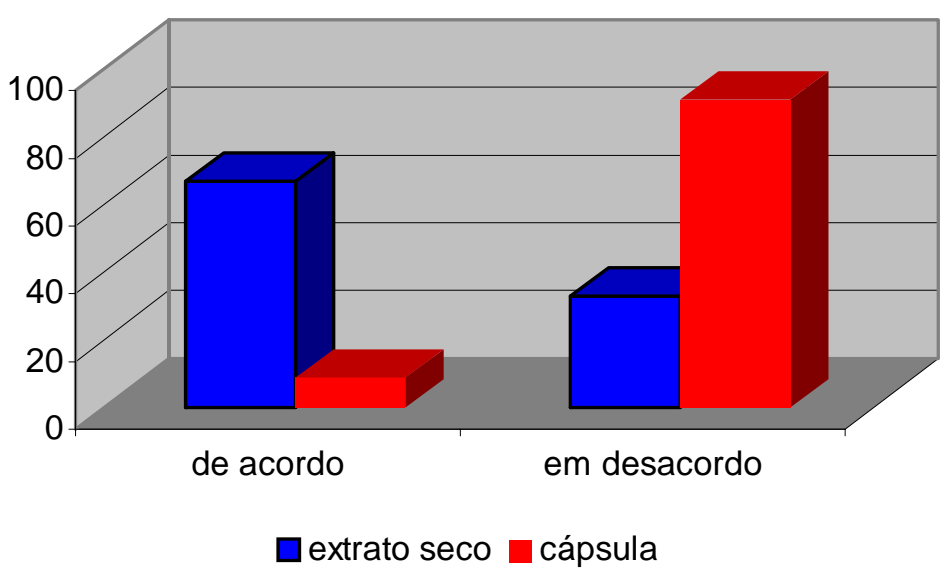

Figura 3. Variação em percentual dos teores (método CLAE) de ginkgoflavonóides, comparados ao valor rotulado de matérias primas e fitoterápicos a base de Ginkgo biloba.

O uso de extratos secos nas formulações farmacêuticas apresenta vantagens em relação ao uso da planta em pó, tais como uma maior concentração de princípios ativos, a possibilidade de padronização do conteúdo de princípios ativos, melhorar a farmacocinética e menor contaminação microbiana. Entretanto, apresenta desvantagens de uso por serem muito higroscópicos e por possuírem custos maiores (CRIPPA, 1978; PETROVICK, et al, 1997).

Os resultados encontrados neste trabalho permitem sugerir que as matérias primas analisadas e que estavam em desacordo com as especificações poderiam, provavelmente, estar com teor de umidade acima de 5\%, recomendado pela FARMACOPÉIA BRASILEIRA (1959). Para prevenir este problema sugere-se o uso de dessecantes, na forma de sílica, nas embalagens de armazenamento, no almoxarifado das farmácias ou armazenamento em dessecadores e o uso em ambientes com umidade controlada. No caso das cápsulas, além desta interferência, devem ser revistos os protocolos de produção das mesmas. 
Tabela 1. Determinação de ginkgoflavonóides em matérias primas e fitoterápicos

\begin{tabular}{|c|c|c|}
\hline Amostra & Fabricante $^{4}$ & $\begin{array}{c}\text { Teor de } \\
\text { ginkgoflavonóides }{ }^{1} \text { ou } \\
\text { Dosagem }^{2}\end{array}$ \\
\hline \multicolumn{3}{|c|}{ Extrato padronizado (no mínimo $24,0 \%$ ) } \\
\hline 1 & $\mathrm{~A}$ & 22,09 \\
\hline 2 & A & 27,94 \\
\hline 3 & A & 24,00 \\
\hline 4 & A & 24,02 \\
\hline 5 & B & 20,06 \\
\hline 6 & $\mathrm{C}$ & 19,69 \\
\hline 7 & A & 25,87 \\
\hline 8 & A & 28,59 \\
\hline 9 & A & 23,70 \\
\hline 10 & A & 28,94 \\
\hline 11 & $\mathrm{D}$ & 22,98 \\
\hline 12 & $\mathrm{E}$ & 15,89 \\
\hline 13 & A & 24,48 \\
\hline 14 & A & 22,37 \\
\hline 15 & A & 20,73 \\
\hline \multicolumn{3}{|c|}{ Cápsulas (concentração 80,0 mg) } \\
\hline 1 & $\mathrm{~A} / 1^{\mathrm{b}}$ & 70,24 \\
\hline 2 & $A / 2$ & 100,8 \\
\hline 3 & $\mathrm{~A} / 3$ & 87,4 \\
\hline 4 & A / 4 & 114,5 \\
\hline 5 & A/5 & 88,4 \\
\hline 6 & $E / 6$ & 75,94 \\
\hline 7 & A / 7 & 102,6 \\
\hline 8 & $\mathrm{D} / 8$ & 80,15 \\
\hline 9 & A/9 & 109,02 \\
\hline 10 & $\mathrm{~A} / 10$ & $-{ }^{3}$ \\
\hline 11 & $\mathrm{~B} / 11$ & 0,73 \\
\hline
\end{tabular}

1. teor encontrado nas matérias primas analisadas

2. concentração $(\mathrm{mg})$ nos produtos acabados analisados

médio

3. em desacordo com as especificações farmacopêicas (FARMACOPÉIA BRASILEIRA, 1988) para peso

4. todas as amostras estavam dentro do prazo de validade; algumas matérias-primas pertenciam ao mesmo lote de produção

5. fabricante da matéria prima / fabricante do produto acabado

\section{CONCLUSÕES}

A técnica CLAE constitui um sistema eficiente para a separação de muitas misturas complexas. O método reportado por STICHER (1993) e aplicada neste estudo, com algumas modificações, mostrou ser simples, rápido e adequado para a análise de matérias primas e de fitoterápicos a base de Ginkgo biloba.

Esta avaliação preliminar foi de grande importância no sentido de indicar parâmetros de qualidade de produtos farmacêuticos constituídos por Ginkgo. Um estudo complementar envolvendo desenvolvimento, aplicação e validação de outras metodologias e outras formas farmacêuticas está sendo realizado neste laboratório.

\section{REFERÊNCIAS BIBLIOGRÁFICAS}

BAST, A.; CHANDLER, R.F.; CHOY, P.C.; DELMULLE, L.M.; GRUENWALD, J.; HALKES, S.B.A.; KELLER, K.; KOEMAN, J.H.; PETER, P.; PRZYREMBEL, H.; REE, E.M.; RENWICK, A.G.; VERMEER, I.T.M. Botanical health 
products, positioning and requirements for effective and safe use. Environmental Toxicology and Pharmacology. $v$. 12, p. 195-211, 2002.

BAUER, R. Quality criteria and standardization of phytopharmaceuticals: can acceptabçle drug standards be achieved? Drug Information Journal. v.32, p. 101-110, 1998.

BAUER, R.; TITTEL, G. Quality assessment of herbal preparations as a precondition of pharmacological and clinical studies. Phytomedicine. v. 2, n. 3, p. $193-198,1996$.

BRASIL. Ministério da Saúde. Agência Nacional de Vigilância Sanitária, Resolução - RE nº 89, de 16 de março de 2004.Lista de Registro Simplificado de Fitoterápicos. Brasília, 2004.

CAPASSO, R.; IZZO, A.A.; PINTO, L.; BIFULCO, T.; VITOBELLO, C.; MASCOLO, N. Phytotherapy and quality of herbal medicines. Fitoterapia, v. 71, p. S58-S65, 2000.

CALIXTO, J.B. EFFICACY, Safety, quality control, marketing and regulatory guidelines for herbal medicines (Phytotherapeutic agents). Brazilian Journal of Medical and Biological Research. v. 33, p.179-189. 2000.

CHOI, D.W.; KIM, J.H.; CHO, S.Y.; HIM, D.H.; CHANG, S.Y. Regulation and quality control of herbal drugs in Korea. Toxicology v. 181-182, p. 581-586. 2002.

CRIPPA, F. Problems of Pharmaceutical Technics with Plant Extracts. Fitoterapia. v. 49, p.257-263, 1978.

DENG, F.; ZITO, S.W. Development and validation of a gas chromatographic-mass spectrometric method for simultaneous identification and quantification of marker compounds uncluding bilobalide, ginkgolides and flavonoids in Ginkgo biloba L. extract and pharmaceutical preparations. Journal of Chromatography A. v. 986, n. 1, p. 121-127, 2003.

DESAI, A.K.; GROSSBERG, G.T. Herbals and botanicals in geriatric psychiatry. American Journal of Geriatric and Psychiatry. v. 11, n. 5, p. 498-506, 2003.FARMACOPÉIA BRASILEIRA: 4ª. ed. São Paulo: Editora Atheneu, parte 1, 1988

KRESSMANN, S.; BIBER, A.; WONNEMANN, M.; SCHUG, B.; BLUME, H.H.; MULLER, W.E. Influence of pharmaceutical quality on the bioavailabidlity of active components from Ginkgo biloba preparations. Journal of Pharmaceutical Pharmacology. v. 54, n. 11, p. 1507-1514. 2002.

LOEW, D. Is the biopharmaceutical quality of extracts adequate for clinical pharmacology? International Journal of Clinical Pharmacology and Therapeutics. V. 35, n.7, p. 302-306, 1997.

PETROVICK, P.R.; ORTEGA, G.G.; BASSANI, V.L.. From a medicinal plant to a pharmaceutical dosage form. A (still) long way for the Brazilian medicinal plants. Ciência e Cultura. v. 49, n. 5/6, p. 364-368, 1997.

SIMÕES, C.M.O.; FARIAS, M.R. Equivalência terapêutica entre medicamentos fitoterápicos. Folha Médica. v. 121, n. 2, p. 65-71. 2002.

STICHER, O. Quality of Ginkgo preparations. Planta Medica. v. 59, p. 2-11, 1993.

SUZUKI, S.F. O Mercado de Medicamentos Fitoterápicos no Brasil. In: SCHULZ, V.; HANSEL, R.; TYLER, V.E. Fitoterapia Racional. São Paulo: Editora Manole Ltda. 2002

VAN BEEK, T.A.; WINTERMANS, M.S. Preparative isolation and dual column high-performance liquid chromatography of ginkgolic acids from Ginkgo biloba. Journal of Chromatography A. v.930, n.1-2, p. 109-117. 2001.

UNITED STATES PHARMACOPEIA - The National Formulary - 25th edithion, NF 20, S2, Rochville, 2002, CDROM.

YUNES, R.A.; PEDROSA, R.C.; CECHINEL FILHO, V. Fármacos e fitoterápicos: a necessidade do desenvolvimento da indústria de fitoterápicos e fitofármacos no Brasil. Química Nova. v. 24, n.1, p. 147-152, 2001. 
WANG, F.M.; YAO, T.W.; ZENG, S. Disposition of quercetin and kaempferol in human following an oral administration of Ginkgo biloba extract tablets. European Journal of Drug Metabolism and Pharmacokinetics. v. 28, n. 3, p. 173-177, 2003.

WATSON, D.G.; OLIVEIRA, E.J. Solid-phase extraction and gas chromatography-mass spectrometry determination of kaempferol and quercetin in human urine after consumption of Ginkgo biloba tablets. Journal of Chromatography B Biomedical Science Applied. v. 723, n. 1-2, p. 203-210, 1999.

WATSON, D.G.; PITT, A.R. Analysis of flavonoids in tablets and urine by gas chromatography/mass spectrometry and liquid chromatography /mass spectrometry. Rapid Communication Mass Spectrometry. v. 12, n. 4, p. 153-156, 1998.

WILLIAMSON, E. M. Synergy and other interactions in phytomedicines. Phytomedicine. v. 8. n. 5, p. 401-409. 2001. 